\title{
OHBEFS \\ Drink coffee or tea to reduce risk of type 2 diabetes mellitus?
}

Drinking coffee or tea could reduce a person's risk of developing type 2 diabetes mellitus (T2DM).

A research group from Boston used data from the Nurses' Health Study (NHS) and the Health Professionals Follow-up Study (HPFS) to investigate the relationship between beverage intake and T2DM risk. The NHS took place between 1984 and 2008 , and was a cohort study involving 121,701 US women who were aged 30-55 years at the time of enrollment. The HPFS, which took place between 1986 and 2008 in the USA, was a prospective study involving 51,529 men who were enrolled aged 40-75 years. In both studies, food and beverage intake were ascertained using food-frequency questionnaires that were sent out every 4 years. The investigators in the current study used data from both the NHS and the HPFS to calculate patient-years from receipt of a baseline questionnaire to self-reported T2DM diagnosis, death or end of follow-up.
In agreement with a number of other studies, the team found that intake of either caffeinated or decaffeinated sugarsweetened beverages was associated with an increased risk of developing T2DM. Additionally, they found a small nonsignificant association between intake of caffeinated and decaffeinated artificially sweetened beverages and T2DM risk in men. However, intake of either caffeinated or decaffeinated coffee was associated with a reduction in T2DM risk. The risk was reduced $4-8 \%$ for every 1-cup increment in daily coffee intake. In women, caffeinated tea intake was also associated with a reduction in T2DM risk.

The researchers also estimated the effect of replacing intake of one type of beverage with another. They estimated that replacement of caffeinated sugarsweetened or artificially sweetened beverages with caffeinated coffee or tea would reduce the risk of developing T2DM. Their estimates also suggest

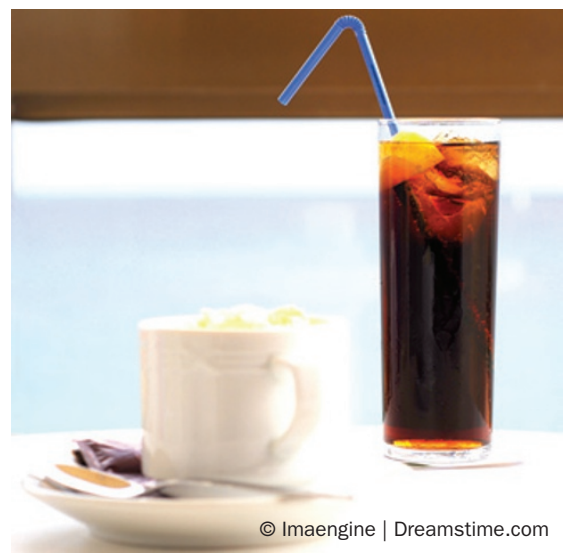

that replacing decaffeinated carbonated beverages with decaffeinated coffee reduces T2DM risk.

The positive effect of coffee intake on T2DM risk was independent of its caffeine content. The authors suggest that antioxidants in these drinks could influence T2DM risk.

Fiona Mitchell

Original article Bhupathiraju, S. N. et al. Caffeinated and caffeine-free beverages and risk of type 2 diabetes. Am. J. Clin. Nutr. doi:10.3945/ajcn.112.048603 